\title{
The effect of step-wised protocol on critical care management of severe aneurysmal subarachnoid hemorrhage
}

\author{
Xin $\mathrm{QU}^{1 \#}$, Feng SHANG ${ }^{1 *}$, Yueqiao XU ${ }^{1}$, Weitao $\mathrm{CHENG}^{1}$, Wenjin $\mathrm{CHEN}^{1}$, Meng $\mathrm{QI}^{1}$, Lidan JIANG ${ }^{1}$, Ning WANG ${ }^{1 *}$ (D)
}

\begin{abstract}
To explore the critical care treatment and clinical outcome of patients with severe aneurysmal subarachnoid hemorrhage (SaSAH), and to provide clinical evidence for the treatment of patients with SaSAH. 160 patients with SaSAH of Hunt and Hess $(\mathrm{H} \& \mathrm{H})$ grade III or above in the neurosurgical critical care unit at ourospital between June 2016 and June 2018 were enrolled in this study. Patients were treated with brain protective bundle based on step-wised protocol under multimodal monitoring and followed up for 3 months. The incidence of various clinical complications and the clinical prognosis after discharge were statistically analyzed. Furthermore, it carried on the correlation analysis to the risk factors and the prognosis. In 160 patients with SaSAH, 127 patients had responsible aneurysms located in the anterior circulation, and the other 33 patients were located in the posterior circulation. 70 patients (43.8\%) underwent transcranial clipping, 81 patients $(50.6 \%)$ underwent endovascular embolization and 9 patients (5.6\%) did not intervene. Patients with IV and V grades of Glasgow Outcome Scale (GOS) were considered good recovery, and those with grade I, II and III were poor prognosis. The 30 -day good prognosis rate was $41.3 \%$ $(66 / 160)$ and the poor prognosis rate was 58.7\% (94/160). Multivariate logistic regression analysis showed that the Hunt \& Hess classification correlated well with prognosis (OR: 4.367,95\% CI, 2.254-8.460, $\mathrm{P}<0.001$ ). The clinical prognosis of patients with $\mathrm{SaSAH}$ is still poor. In particular, under the critical care of neurosurgery, the application of brain protective bundle based on step-wised protocol under multimodal monitoring might improve the clinical prognosis of patients with SaSAH.
\end{abstract}

Keywords: severe aneurysmal subarachnoid hemorrhage; multimodal monitoring; step-wise bundled strategy; complications; prognosis

Practical Application: Our results suggested that under the critical care of neurosurgery, the application of brain protective bundle based on step-wised protocol under multimodal monitoring might improve the clinical prognosis of patients with SaSAH.

\section{Introduction}

Aneurysmal subarachnoid hemorrhage (aSAH) is a common clinical disease with an annual incidence of about 1/10000 (Steiner et al., 2013). Despite the state-of-the-art treatment given to patients, the 30 -day mortality rate is still as high as $36 \%-42 \%$, while about $25 \%-50 \%$ of the survivors remain disabled (Lawton \& Vates 2017; Connolly et al., 2012). The clinical outcomes of patients depend on a variety of factors, including the severity of acute bleeding, the initial condition of the patient, the presence of early rebleeding, and the presence or absence of delayed cerebral ischemia (DCI) (Kundra et al., 2014). Lung and heart complications are also associated with prognosis (D'Souza, 2015). Studies have shown that prognosis is associated with poor clinical performance and delayed cerebral ischemia. Sejkorová et al. (2016) considered an initially high Hunt and Hess (H\&H) grade Hunt \& Hess, 1968) as an unfavorable factor for the clinical outcome of patients with ruptured aneurysms of the posterior inferior cerebellar artery after interdisciplinary treatment.

With the development of microsurgical operative techniques and endovascular embolization techniques, the mortality and disability rate of patients with low-grade aneurysms have decreased significantly (Molyneux et al., 2005). However, perioperative treatment of severe aneurysmal subarachnoid hemorrhage (SaSAH) is still challenging due to early brain

injury, secondary nerve damage, and systemic complications. In our study, we aimed to retrospectively analyzed the clinical data of patients with SaSAH and the risk factors affecting the prognosis after treated with brain protective bundle based on step-wised protocol under multimodal monitoring.

\section{Patients and methods}

\subsection{Patients}

This study was approved by the Ethics committee of our hospital. A total of 186 patients with SaSAH of Hunt and Hess $(\mathrm{H} \& \mathrm{H})$ grade III or above in the neurosurgical critical care unit at our hospital between June 2016 and June 2018 participated in in this study. We retrospectively analyzed the clinical data of 160 eligible patients, including 68 males and 92 females. All patients signed the informed consent.

Inclusion criteria: (1) Acute inpatients within $72 \mathrm{~h}$ after onset, with sudden headache and disturbance of consciousness as the main clinical manifestations. (2) The patient was diagnosed as SAH by brain computed tomography (CT) and confirmed as aSAH by digital subtraction angiography (DSA) or computed tomography angiography (CTA). (3) Clinical evaluation of 
H\&H was grade III and above (Hunt \& Hess, 1968) Exclusion criteria: (1) Patients with negative DSA diagnosis. (2) Patients with $\mathrm{H} \& \mathrm{H}$ grade $\mathrm{V}$ had deep coma, bilateral mydriasis and no spontaneous respiration.

\subsection{The critical care of neurosurgery}

According to the clinical classification, patients were performed multimodal neurocritical monitoring, including vital signs, electroencephalogram, bispectral index (BIS), intracranial pressure (ICP) and transcranial doppler (TCD) (Ko, 2013). The application of brain protective bundle based on step-wised protocol under multimodal monitoring was performed to treat patients (Lee et al., 2014; Green et al., 2013; Magni et al., 2015). According to the results of $\mathrm{H} \& \mathrm{H}$ classification and multimodal monitoring, the patients with $\mathrm{H} \& \mathrm{H}$ grade III, mild brain injury and moderate increase of ICP were controlled by body position, sedation and analgesia, lumbar puncture or external drainage of lumbar cistern. For the patients with H\&H grade III and IV, severe brain injury and moderate to severe increase of intracranial pressure, secondary control methods such as ICP monitoring or external ventricular drainage, osmotic dehydration drugs and improvement of colloidal osmotic pressure should be used on the basis of the first grade. In addition to the primary and secondary control methods, the patients with $\mathrm{H} \& \mathrm{H}$ grade IV and V, severe brain injury and severe increase of ICP were controlled by systemic temperature to maintain core temperature of $34^{\circ} \mathrm{C}-36^{\circ} \mathrm{C}$, sedation depth of BIS $40-50$, and strict blood pressure management to maintain cerebral perfusion pressure (CPP) of 60-80 mmHg.

\subsection{Determination of clinical complications}

Diagnosis standard of cerebral vasospasm: according to the clinical symptoms of nervous system, new cerebral infarction and aneurysmal rerupture and bleeding were excluded. Combined with the monitoring results of continuous TCD, it showed that the average blood flow velocity of middle cerebral artery and anterior cerebral artery was more than $120 \mathrm{~cm} / \mathrm{s}$, or the average blood flow velocity of posterior cerebral artery was more than $90 \mathrm{~cm} / \mathrm{s}$, and gradually increased. Pneumonia, abnormal liver function, hypoproteinemia, anemia and electrolyte disorder were determined by the deviation from the normal value according to the diagnostic standard of internal medicine.

\subsection{Follow-up}

Glasgow Outcome Scale (GOS) was used for clinical follow-up evaluation. 3 months after discharge, the patients were followed up in the outpatient department or their family members were contacted by telephone for scoring. Patients with grade IV and $\mathrm{V}$ of GOS had a good prognosis, while patients with grade I to III of GOS had a bad prognosis.

\subsection{Statistical analysis}

SPSS software (version 18.0) was used for statistical analysis. Measurement data are presented as means \pm SD. Numeration data are presented as percentage. The measurement data and numeration data were statistically analyzed with $t$ test and $\chi^{2}$ test respectively. Single factor and multi factor Logistic regression analysis were used to screen the influencing factors related to GOS grade of prognosis. $\mathrm{P}<0.05$ was considered to be statistically different.

\section{Results}

\subsection{The general clinical data}

There were 160 patients who met the inclusion criteria included in our study, including 68 males and 92 females. The patients aged from 24 to 87 years, with an average age of $58.7 \pm 11.9$ years. Among them, $101(63.1 \%)$ had hypertension, $12(7.5 \%)$ had diabetes, and $22(13.8 \%)$ had coronary heart disease. In 160 patients with SaSAH, 127 patients had responsible aneurysms located in the anterior circulation, and the other 33 patients were located in the posterior circulation. According to the patient's condition and DSA results, the neurosurgery and the cerebrovascular interventional therapy physicians selected surgical clipping or endovascular interventional embolization of aneurysms for patients within $24 \mathrm{~h}$ after admission. Among them, 70 patients (43.8\%) underwent transcranial clipping, 81 patients (50.6\%) underwent endovascular embolization, and 9 patients (5.6\%) did not intervene. Because of the location and shape of the aneurysm, systemic diseases and the wishes of the family members, those 9 patients were not treated and only critical care was performed. The results were showed in Table 1.

Table 1. The general clinical data.

\begin{tabular}{|c|c|c|c|c|c|}
\hline \multirow{2}{*}{ Factors } & \multicolumn{3}{|c|}{ H\&H grade } & \multirow[b]{2}{*}{ Test value } & \multirow[b]{2}{*}{$\mathrm{P}$} \\
\hline & III $(n=51)$ & IV $(n=83)$ & $V(n=26)$ & & \\
\hline Ages (means \pm SD) & $58.2 \pm 13.7$ & $56.4 \pm 11.5$ & $60.4 \pm 9.0$ & $0.332^{\mathrm{a}}$ & 0.718 \\
\hline Gender (male/female) & $18 / 33$ & $40 / 43$ & $10 / 16$ & $2.358^{\mathrm{b}}$ & 0.308 \\
\hline \multicolumn{6}{|l|}{ Underlying diseases } \\
\hline Hypertension (\%) & $25(49.0)$ & $58(69.9)$ & $18(69.2)$ & $6.402^{\mathrm{b}}$ & 0.041 \\
\hline Diabetes mellitus (\%) & $1(2.0)$ & $7(8.4)$ & $4(15.4)$ & $4.690^{\mathrm{b}}$ & 0.096 \\
\hline Coronary heart disease (\%) & $7(13.7)$ & $8(9.6)$ & $7(26.9)$ & $4.987^{b}$ & 0.083 \\
\hline Responsible aneurysm site & & & & $4.299^{b}$ & 0.367 \\
\hline Anterior circulation [n (\%)] & $44(86.3)$ & $63(75.9)$ & $20(76.9)$ & & \\
\hline Posterior circulation [n (\%)] & $7(13.7)$ & $20(24.1)$ & $6(23.1)$ & & \\
\hline
\end{tabular}

${ }^{\mathrm{a}}$ represents $t$ value, ${ }^{\mathrm{b}}$ represents $\chi^{2}$ value. 


\subsection{Complications in the treatment of SaSAH}

The main complications of patients with SaSAH were pulmonary infection, electrolyte disorder, hypoproteinemia, anemia, cerebral vasospasm, cerebral infarction and liver dysfunction. As shown in Table 2, the complications of nervous system and other systems in patients with SaSAH were increased with the increase of $\mathrm{H} \& \mathrm{H}$ grade, and the incidence of complications in each grade group was significantly different.

\subsection{Follow-up results and related factors analysis}

The 3-months good prognosis rate of patients with SaSAH was $41.3 \%(66 / 160)$ and the poor prognosis rate was $58.7 \%$ (94/160). The average hospitalization days and 3-months prognosis of patients with different $\mathrm{H} \& \mathrm{H}$ grades were shown in Table 3. Univariate analysis of age (65 years old as the demarcation point), gender, clinical grade, and various complications as prognostic risk factors, $\mathrm{H} \& \mathrm{H}$ classification, electrolyte imbalance, anemia, hypoproteinemia, and cerebral infarction were related to poor prognosis (Table 4). Multivariate logistic regression analysis showed that the main factor affecting prognosis was Hunt \& Hess classification (Table 5).

\section{Discussion}

$\mathrm{SaSAH}$ is a common clinical disease in neurosurgery, which clinically manifested as sudden and excruciating headache (Lionel et al., 2019). In recent years, with the development of microsurgical operative techniques and endovascular embolization techniques, it has greatly improved the prognosis of patients. However, the mortality and disability rate of aSAH, especially severe aSAH, are still as high as 36\%-42\% (Steiner et al. 2013; Lawton \& Vates, 2017; Connolly et al., 2012). Therefore, the clinical treatment of $\mathrm{SaSAH}$ is still a hot and difficult problem in neurosurgery.

The mortality and outcome after ruptured aneurysm hemorrhage depends on the bleeding location, severity, and clinical intervention (Kundra et al., 2014). The commonly used clinical classification based on the severity of nervous system injury is $\mathrm{H} \& \mathrm{H}$ classification, which takes consciousness and nervous system symptoms and signs as the standard. World Federation of Neurosurgical Societies scale (WFNS) classification was combined

Table 2. Analysis of clinical complications.

\begin{tabular}{|c|c|c|c|c|c|}
\hline \multirow{2}{*}{ Clinical complications } & \multicolumn{3}{|c|}{$\mathrm{H} \& \mathrm{H}$ grade } & \multicolumn{2}{|c|}{ Values } \\
\hline & III $(\mathrm{n}=51)$ & IV $(\mathrm{n}=83)$ & $V(n=26)$ & $x^{2}$ & $\mathrm{P}$ \\
\hline Pulmonary infection [n (\%)] & $25(49.0 \%)$ & $53(63.9 \%)$ & $19(73.1 \%)$ & 4.929 & 0.085 \\
\hline Electrolyte disorder [n (\%)] & $33(64.7 \%)$ & $71(85.5 \%)$ & $21(80.8 \%)$ & 8.152 & 0.017 \\
\hline Hypoproteinemia [n (\%)] & $21(41.2 \%)$ & $51(61.4 \%)$ & $22(84.6 \%)$ & 13.926 & 0.001 \\
\hline Anemia [n (\%)] & $19(37.3 \%)$ & $44(53.0 \%)$ & $20(76.9 \%)$ & 10.944 & 0.004 \\
\hline Cerebral vasospasm [n (\%)] & $15(29.4 \%)$ & $38(45.8 \%)$ & $8(30.8 \%)$ & 4.301 & 0.116 \\
\hline Cerebral infarction $[\mathrm{n}(\%)]$ & $8(15.7 \%)$ & $23(27.7 \%)$ & $9(34.6 \%)$ & 3.967 & 0.138 \\
\hline Liver dysfunction [n (\%)] & $8(15.7 \%)$ & $29(34.9 \%)$ & $13(50.0 \%)$ & 10.531 & 0.005 \\
\hline
\end{tabular}

Table 3. Clinical prognosis of patients with SaSAH.

\begin{tabular}{|c|c|c|c|c|c|}
\hline \multirow{2}{*}{ Clinical prognosis } & \multicolumn{3}{|c|}{ H\&H grade } & \multicolumn{2}{|c|}{ Values } \\
\hline & III $(n=51)$ & IV $(\mathrm{n}=83)$ & $\mathrm{V}(\mathrm{n}=26)$ & Test value & $\mathrm{P}$ \\
\hline Hospitalization days (means \pm SD) & $13 \pm 9$ & $15 \pm 11$ & $20 \pm 11$ & $3.828^{\mathrm{a}}$ & 0.024 \\
\hline 3-months GOS & & & & $83.033^{b}$ & $<0.001$ \\
\hline $\mathrm{V}[\mathrm{n}(\%)]$ & $28(54.9 \%)$ & $5(6.0 \%)$ & $1(3.8 \%)$ & & \\
\hline IV [n (\%)] & $15(29.4 \%)$ & $15(18.1 \%)$ & $2(7.7 \%)$ & & \\
\hline III [n (\%)] & $5(9.8 \%)$ & $41(49.4 \%)$ & $12(46.2 \%)$ & & \\
\hline II $[\mathrm{n}(\%)]$ & $2(3.9 \%)$ & $17(20.5 \%)$ & $3(11.5 \%)$ & & \\
\hline $\mathrm{I}[\mathrm{n}(\%)]$ & $1(2.0 \%)$ & $5(6.0 \%)$ & $8(30.8 \%)$ & & \\
\hline
\end{tabular}

${ }^{\text {a }}$ represents $t$ value, ${ }^{\mathrm{b}}$ represents $\chi^{2}$ value.

Table 4. Univariate regression analysis of clinical prognosis of GOS grade.

\begin{tabular}{lcc}
\hline Variable & Test value & P \\
\hline Ages & 7.310 & 0.120 \\
Gender & 6.606 & 0.158 \\
Cerebral infarction & 14.511 & $0.006^{*}$ \\
Cerebral vasospasm & 5.238 & 0.264 \\
Pulmonary infection & 5.777 & 0.216 \\
Hypoalbuminemia & 9.574 & $0.048^{*}$ \\
Anemia & 15.917 & $0.003^{*}$ \\
Electrolyte disorder & 14.879 & $0.005^{*}$ \\
H\&H grade & 61.748 & $<0.001^{*}$ \\
\hline
\end{tabular}

The test value was chi square; ${ }^{\star} \mathrm{P}<0.05$, the difference was statistically significant. 
Table 5. Multivariate regression analysis of clinical prognosis of GOS grade.

\begin{tabular}{lccc}
\hline Variable & OR & $95 \%$ CI & P \\
\hline Cerebral infarction & 1.822 & $0.720-4.608$ & 0.205 \\
Hypoalbuminemia & 0.635 & $0.290-1.393$ & 0.257 \\
Anemia & 0.570 & $0.261-1.241$ & 0.157 \\
Electrolyte disorder & 0.415 & $0.166-1.036$ & 0.059 \\
H\&H grade & 4.367 & $2.254-8.460$ & $<0.001^{*}$ \\
\hline
\end{tabular}

${ }^{*} \mathrm{P}<0.05$, the difference was statistically significant.

with GCS coma score (Rosen \& MacDonald, 2005). Although they are slightly different, they have a good predictive value for the prognosis of patients. The higher the grade, the worse the prognosis. The patients in H\&H grade or WFNS grade IV and $\mathrm{V}$ are called high score grade or poor prognosis grade in clinical practice, which are different from the patients in grade I, II and III. According to our clinical experience, patients with grade I and II have less bleeding, less primary and secondary brain injury, and no significant clinical symptoms. After the treatment of aneurysms, the course of disease is short and the prognosis is good. They are low-grade aneurysms. The change of conscious state in patients with grade III indicates that aSAH has affected the function of the whole brain to a certain extent, and the condition is in a changeable state. The proportion of patients in this grade is relatively large, and the adverse prognosis of grade III aneurysm reported from abroad is as high as $52.6 \%$ (Steiner et al. 2013). Some foreign scholars also classified this kind of patients as high-grade aneurysms (Lee et al., 2014). In this study, the 30-day poor prognosis rate of patients with $\mathrm{H} \& \mathrm{H}$ grade III was $15.7 \%$. It is reasonable to call $\mathrm{H} \& \mathrm{H}$ grade III and above as "subarachnoid hemorrhage of aneurysmal severity" from the perspective of improving patients' prognosis and strengthening management, and carry out critical care management (Lee et al., 2014; Petridis et al., 2017).

Another factor to be noted is that the age of high incidence of aSAH is over 50 years old. The serious basic diseases such as coronary heart disease, hypertension and diabetes in the elderly also affect the clinical outcome of the patients. For this population, we should increase the first grade score and strengthen the monitoring and treatment according to the patients with poor prognosis, which is conducive to improving the clinical prognosis of these patients. In this study, univariate analysis and multifactor analysis of patients showed that clinical classification was still the main influencing factor of prognosis.

The main goal of the treatment of aneurysm itself is to prevent the rupture of aneurysm. Therefore, whether it is surgical clipping or endovascular embolization, the common treatment consensus is that the treatment should be carried out in the early stage. At the same time, the treatment of aneurysms provides the necessary basis for the sequential inertial intensive care. Especially for high-grade patients, the invasive interventions such as extraventricular drainage, intracranial pressure monitoring, lumbar puncture or lumbar cistern drainage, respiratory and circulatory support are more safe because of the brain injury and systemic complications caused by the rupture of aneurysms. In this study, the patients were all operated on aneurysm within 72 hours after the onset of the disease, which made it possible to carry out brain protective bundle based on step-wised protocol under multimodal monitoring and treatment after the operation. In this study, endovascular embolization and surgical operation accounted for half of the patients. However, the embolization treatment for the patients with grade III and V was more than surgical clipping, which may be related to the relatively light symptoms of patients with grade III. The treatment with less trauma was more easily accepted by patients. While the symptoms in patients with grade $\mathrm{V}$ were more serious, and surgical clipping had higher requirements for anesthesia and surgical technology. The patients with grade IV chose more surgery, and more consideration was given to the possibility of external decompression. This study does not carry on the correlation analysis to the treatment methods, and it is one of the shortcomings.

After the rupture of aneurysm, in addition to the early brain injury caused by the location and amount of bleeding (Fujii et al., 2013), various secondary pathological factors, such as rebleeding, DCI, hydrocephalus, complications related to long-term bed rest, stress hyperglycemia, cardiopulmonary complications and hypercoagulable state, make clinical treatment need to carry out multimodal monitoring and intervention (Veldeman et al., 2016; Garry et al., 2016; Meguro et al., 2016; Francoeur \& Mayer, 2016) At present, the treatment of early brain injury, such as subarachnoid hemorrhage which is mainly characterized by the increase of ICP, brain injury and brain edema, is usually based on the patient's condition and monitoring results to carry out step-by-step intervention (Stocchetti \& Maas 2014). In clinic, this control method of brain injury has become a standardized treatment. However, for severe patients, the progressive control of intracranial pressure and brain injury is not conducive to limiting the progress of early brain injury. All the patients in this study were intervened in the early stage of the disease to reduce the risk of aneurysm rupture. Based on the results of multimodal monitoring and the clinical classification of patients, we have carried out the control and treatment of brain injury (including temperature control) on the basis of step by step measures. The purpose of this treatment was to reduce the peak of early brain injury in the peak of pathological changes of the disease, and improve the prognosis of patients (Choi et al., 2017). According to the severity and the different stages of the course of the disease, the brain protective bundle based on step-wised protocol under multimodal monitoring may be a way to improve the clinical prognosis of patients.

Because of the direct effect of severe brain injury on the whole body after aSAH, there are many systemic complications in severe patients, and serious systemic complications, such as lung infection and respiratory failure, will not only cause shock, but also cause hypoxemia, which are part of the secondary injury and affect the recovery of nerve function. In this study, cerebral 
infarction, cerebral vasospasm, abnormal liver function and other complications occurred in patients, with the highest incidence of pulmonary infection, anemia, and electrolyte disorder. In this study, univariate analysis suggested that complications of pulmonary infection, anemia and electrolyte disorder were related to prognosis, while multivariate analysis showed that only $\mathrm{H} \& \mathrm{H}$ classification; Hess grade was related to prognosis. From our clinical experience, systemic complications in modern intensive care treatment less directly affect the prognosis of patients, which reflects the advantages of critical care. In this study, with the increase of clinical grade, the length of stay and the cost of medical treatment were also increased. Therefore, it is necessary to intervene many secondary pathological states in order to improve the prognosis.

There are also some limitations in our study. Firstly, the diagnosis standard of internal medicine was used to determine the complications during the treatment of ICU. The disadvantage is that there is no stratification of the severity, and the influence weight of different severity on the prognosis is different. Secondly, the mortality and disability rate of patients in this study is lower than that of the previous multicenter study (Zhao et al., 2014), which may be related to the annual admission capacity of single center patients and the strong homogeneity of intensive care treatment level. Although patients with severe aneurysms are classified by grade, the different location and size of aneurysms and the methods of clinical intervention, such as embolization or clipping, may be the factors affecting the prognosis of patients. The follow-up time in this study was 3 months after discharge, and some complications, such as secondary hydrocephalus, needed at least 3-6 months to observe. The lack of long-term prognosis follow-up results was also a defect.

In conclusion, our results suggested that under the critical care of neurosurgery, the application of brain protective bundle based on step-wised protocol under multimodal monitoring might improve the clinical prognosis of patients with SaSAH. In the future, it needs to be verified by prospective randomized controlled study with routine severe surveillance treatment.

\section{References}

Choi, W., Kwon, S. C., Lee, W. J., Weon, Y. C., Choi, B., Lee, H., Park, E. S., \& Ahn, R. (2017). Feasibility and safety of mild therapeutic hypothermia in poor-grade subarachnoid hemorrhage: prospective pilot study. Journal of Korean Medical Science, 32(8), 1337-1344. http://dx.doi.org/10.3346/jkms.2017.32.8.1337. PMid:28665071.

Connolly, E. S. Jr., Rabinstein, A. A., Carhuapoma, J. R., Derdeyn, C. P., Dion, J., Higashida, R. T., Hoh, B. L., Kirkness, C. J., Naidech, A. M., Ogilvy, C. S., Patel, A. B., Thompson, B. G., Vespa, P., American Heart Association Stroke Council, Council on Cardiovascular Radiology and Intervention, Council on Cardiovascular Nursing, Council on Cardiovascular Surgery and Anesthesia, \& Council on Clinical Cardiology. (2012). Guidelines for the management of aneurysmal subarachnoid hemorrhage: a guideline for healthcare professionals from the American Heart Association/american Stroke Association. Stroke, 43(6), 1711-1737. http://dx.doi.org/10.1161/ STR.0b013e3182587839. PMid:22556195.

D'Souza, S. (2015). Aneurysmal subarachnoid hemorrhage. Journal of Neurosurgical Anesthesiology, 27(3), 222-240. http://dx.doi. org/10.1097/ANA.0000000000000130. PMid:25272066.
Francoeur, C. L., \& Mayer, S. A. (2016). Management of delayed cerebral ischemia after subarachnoid hemorrhage. Critical Care (London, England), 20(1), 277. http://dx.doi.org/10.1186/s13054-016-1447-6. PMid:27737684.

Fujii, M., Yan, J., Rolland, W. B., Soejima, Y., Caner, B., \& Zhang, J. H. (2013). Early brain injury, an evolving frontier in subarachnoid hemorrhage research. Translational Stroke Research, 4(4), 432-446. http://dx.doi.org/10.1007/s12975-013-0257-2. PMid:23894255.

Garry, P. S., Rowland, M. J., Ezra, M., Herigstad, M., Hayen, A., Sleigh, J. W., Westbrook, J., Warnaby, C. E., \& Pattinson, K. T. (2016). Electroencephalographic response to sodium nitrite may predict delayed cerebral ischemia after severe subarachnoid hemorrhage. Critical Care Medicine, 44(11), e1067-e1073. http://dx.doi.org/10.1097/ CCM.0000000000001950. PMid:27441898.

Green, D. M., Burns, J. D., \& Defusco, C. M. (2013). ICU management of aneurysmal subarachnoid hemorrhage. Journal of Intensive Care Medicine, 28(6), 341-354. http://dx.doi.org/10.1177/0885066611434100. PMid:22328599.

Hunt, W.E., \& Hess, R. M. (1968). Surgical risk as related to time of intervention in the repair of intracranial aneurysms. Journal of Neurosurgery, 28(1), 14-20. http://dx.doi.org/10.3171/jns.1968.28.1.0014. PMid:5635959.

Ko, S.-B. (2013). Multimodality monitoring in the neurointensive care unit: a special perspective for patients with stroke. Journal of Stroke, 15(2), 99-108. http://dx.doi.org/10.5853/jos.2013.15.2.99. PMid:24324945.

Kundra, S., Mahendru, V., Gupta, V., \& Choudhary, A. K. (2014). Principles of neuroanesthesia in aneurysmal subarachnoid hemorrhage. Journal of Anaesthesiology, Clinical Pharmacology, 30(3), 328-337. http:// dx.doi.org/10.4103/0970-9185.137261. PMid:25190938.

Lawton, M. T., \& Vates, G. E. (2017). Subarachnoid hemorrhage. The New England Journal of Medicine, 377(3), 257-266. http://dx.doi. org/10.1056/NEJMcp1605827. PMid:28723321.

Lee, K., Choi, H. A., Edwards, N., Chang, T., \& Sladen, R. N. (2014). Perioperative critical care management for patients with aneurysmal subarachnoid hemorrhage. Korean Journal of Anesthesiology, 67(2), 77-84. http://dx.doi.org/10.4097/kjae.2014.67.2.77. PMid:25237442.

Lionel, K. R., Sethuraman, M., Abraham, M., Vimala, S., Prathapadas, U., \& Hrishi, A. P. (2019). Effect of pregabalin on perioperative headache in patients with aneurysmal subarachnoid hemorrhage: a randomized double-blind, placebo-controlled trial. Journal of Neurosciences in Rural Practice, 10(3), 438-443. http://dx.doi. org/10.1055/s-0039-1697871. PMid:31602153.

Magni, F., Pozzi, M., Rota, M., Vargiolu, A., \& Citerio, G. (2015). Highresolution intracranial pressure burden and outcome in subarachnoid hemorrhage. Stroke, 46(9), 2464-2469. http://dx.doi.org/10.1161/ STROKEAHA.115.010219. PMid:26243224.

Meguro, T., Tanabe, T., Muraoka, K., Terada, K., Hirotsune, N., \& Nishino, S. (2016). Endovascular treatment for aneurysmal subarachnoid hemorrhage with neurogenic pulmonary edema in the acute stage. Turkish Neurosurgery, 26(6), 849-853. PMid:27560529.

Molyneux, A. J., Kerr, R., Yu, L. M., Clarke, M., Sneade, M., Yarnold, J. A., \& Sandercock, P., \& International Subarachnoid Aneurysm Trial (ISAT) Collaborative Group. (2005). International Subarachnoid Aneurysm Trial (ISAT) of neurosurgical clipping versus endovascular coiling in 2143 patients with ruptured intracranial aneurysms: a randomised comparison of effects on survival, dependency, seizures, rebleeding, subgroups, and aneurysm occlusion. Lancet, 366(9488), 809-817. http://dx.doi.org/10.1016/S0140-6736(05)67214-5. PMid:16139655.

Petridis, A. K., Kamp, M. A., Cornelius, J. F., Beez, T., Beseoglu, K., Turowski, B., \& Steiger, H. J. (2017). Aneurysmal subarachnoid hemorrhage. Deutsches Ärzteblatt International, 114(13), 226-236. PMid:28434443. 
Rosen, D. S., \& MacDonald, R. L. (2005). Subarachnoid hemorrhage grading scales: a systematic review. Neurocritical Care, 2(2), 110-118. http://dx.doi.org/10.1385/NCC:2:2:110. PMid:16159052.

Sejkorová, A., Cihlář, F., Hejčl, A., Lodin, J., Vachata, P., \& Sameš, M. (2016). Microsurgery and endovascular treatment of posterior inferior cerebellar artery aneurysms. Neurosurgical Review, 39(1), 159-168, discussion 168. http://dx.doi.org/10.1007/s10143-0150659-6. PMid:26296429.

Steiner, T., Juvela, S., Unterberg, A., Jung, C., Forsting, M., \& Rinkel, G., \& European Stroke Organization (2013). European Stroke Organization guidelines for the management of intracranial aneurysms and subarachnoid haemorrhage. Cerebrovascular Diseases (Basel, Switzerland), 35(2), 93112. http://dx.doi.org/10.1159/000346087. PMid:23406828.
Stocchetti, N., \& Maas, A. I. (2014). Traumatic intracranial hypertension. The New England Journal of Medicine, 370(22), 2121-2130. http:// dx.doi.org/10.1056/NEJMra1208708. PMid:24869722.

Veldeman, M., Hollig, A., Clusmann, H., Stevanovic, A., Rossaint, R., \& Coburn, M. (2016). Delayed cerebral ischaemia prevention and treatment after aneurysmal subarachnoid haemorrhage: a systematic review. British Journal of Anaesthesia, 117(1), 17-40. http://dx.doi. org/10.1093/bja/aew095. PMid:27160932.

Zhao, B., Tan, X., Yang, H., Zheng, K., Li, Z., Xiong, Y., \& Zhong, M., and the AMPAS investigators (2014). A Multicenter prospective study of poor-grade aneurysmal subarachnoid hemorrhage (AMPAS): observational registry study. BMC Neurology, 14(1), 86. http:// dx.doi.org/10.1186/1471-2377-14-86. PMid:24742248. 